\title{
Optimal energy-efficient sensing in cooperative cognitive radio networks
}

\author{
Xia $\mathrm{Wu}^{1,2^{*}}$, Jin-Ling $\mathrm{Xu}^{2}$, Ming Chen ${ }^{2}$ and Jun-Bo Wang ${ }^{2}$
}

\begin{abstract}
In a cognitive radio network (CRN), when the secondary users (SUs) are battery-powered devices, the concept of energy-efficient design is very important. The sensing time and the number of cooperative SUs in the cooperative spectrum sensing could greatly affect energy consumption and throughput of the system. In this paper, we propose an energy utility function by formulating the achievable data rate of a cooperative CRN in terms of the energy consumption by the CRN. The maximization of the energy utility function is obtained by jointly designing the sensing time, sensing threshold, and number of cooperative SUs with the constraint of sufficient protection for primary user (PU). Numerical results show that the CRN can achieve almost the maximum achievable data rate with significant energy saving through the joint optimization.
\end{abstract}

Keywords: Cognitive radio network; Energy-efficient design; Sensing time; Sensing threshold

\section{Introduction}

With the rapid growth of wireless services, scarcity of spectrum resources has become the bottleneck of its development. Under the current fixed spectrum allocation policy, the utilization of the licensed spectrum varies from $15 \%$ to $85 \%$, whereas only $2 \%$ of the spectrum would be used in the US at any given moment [1]. This motivates the advent of cognitive radio network (CRN) which was first introduced in 1999 by J. Mitola III [2], where the secondary users (SUs) opportunistically utilize the temporarily unused spectrum which is licensed to primary user (PU). And CRN has been proved to be an efficient solution for spectrum shortage and underutilization problem.

Up to now, a lot of work has been done to investigate the CNR. In [3], the use of multipath and single-path quality of service (QoS)-aware routing algorithms under harsh smart grid (SG) environmental conditions is investigated in order to evaluate their service differentiation capabilities in reliability and timeliness domains. In [4], a novel approach is proposed to set different reliability values for image packets for image transmission in wireless

*Correspondence: wuxia80@seu.edu.cn

1 Department of Mathematics, Southeast University, 210000 Nanjing, People's Republic of China

2 National Mobile Communications Research Laboratory, Southeast University, 210000 Nanjing, People's Republic of China multimedia sensor networks (WMSNs). Using this prioritization, important parts of an image are assigned high priority and take priority during data transmission. In [5], authors presented opportunities and design challenges of wireless sensor network (WSNs) for smart grid applications. WSN-based smart grid applications have been introduced, and some WSN standards and communication protocols have been discussed for smart grid applications. In [6], the authors proposed a cross-layer framework that employs cognitive radio communication to circumvent the hostile propagation conditions in power systems and supports QoS for smart grid applications. In [7], architectures to support cognitive radio (CR) networks in SG applications, major challenges, and open issues have been discussed.

Compared with traditional communication systems [8,9], CRN requires additional energy consumption, such as the energy used in the sensing process. Furthermore, the accuracy of the sensing results also affects the total energy consumption. Cooperative spectrum sensing could increase the accuracy of the sensing results by mitigating the effect of multipath and shadowing. However, energy consumption is a major concern, in addition to extra sensing time and delay [10], as cooperative spectrum sensing consumes additional energy to report the results to the fusion center.

\section{照 Springer}

C 2014 Wu et al: licensee Springer. This is an Open Access article distributed under the terms of the Creative Commons Attribution License (http://creativecommons.org/licenses/by/4.0), which permits unrestricted use, distribution, and reproduction in any medium, provided the original work is properly credited. 
In this paper, we focus on the energy efficiency because of its importance in a practical CRN which is powered by batteries. We attempt to optimize the parameters which affect the energy consumption and the throughput of the cooperative CRN such as sensing time, sensing threshold, and the number of cooperative SUs. Some recent works dealing with the issues of CRN energy efficiency have been presented in [11-15]. In [11], the authors studied a joint design of energy-efficient sensing and transmission duration for a CRN in which the PU was protected. In [12], the authors defined the energy efficiency as the ratio of the average throughput of the CRN over the average energy used by the CRN and a problem of jointly designing the fusion rule threshold and detector's threshold that maximizes the energy efficiency of the CRN was studied. In [13], the authors proposed a comprehensive utility function by formulating the transmission cost in terms of the energy consumption of sensing process and transmission process. The utility function was maximized by optimizing the sensing time. In [14], the authors identified the sensing-access strategies and the sensing order that achieve the maximum energy efficiency. In [15], a comparison among different decision-fusion rules was studied in terms of the consumed energy and the achievable detection probability at a given false alarm probability threshold.

Motivated by the previous work, we propose an energy utility function by formulating the achievable data rate in terms of the energy consumption in a cooperative CRN. A weighting factor of energy consumption is introduced here. The larger the value of the weighting factor implies that the energy consumption is more important than the achievable data rate of CRN. Taking the maximization of the energy utility as the design objective, the optimization problem is formulated as a function of three variables, which are sensing time, sensing threshold, and the number of cooperative SUs, subject to the target detection probability. An algorithm is provided to solve this problem, and numerical results show that CRN can achieve almost the maximum achievable data rate with significant energy saving through the joint optimization.

The rest of the paper is organized as follows. The system model is presented in the 'System model' section. In the 'Problem formulation' section, we propose an energy utility function and formulate an optimize problem. And we give an algorithm to obtain the solution of the problem in the 'Solution of formulation' section. Numerical results are presented in the 'Numerical results' section. Finally, the 'Conclusions' section draws the conclusion.

\section{System model}

We consider a CRN with $N$ SUs and a fusion center. All the SUs try to use a licensed spectrum (termed channel) without introducing extra interference to the PU of the spectrum. Therefore, the SUs must first perform spectrum sensing to determine the status of the spectrum before using it.

During the sensing time, $M$ out of the $N$ SUs individually sense the status of the channel and make a decision. Then, they send their decisions to the fusion center sequentially. Finally, based on the decisions of the SUs, the fusion center determines the active or idle status of the channel. If the channel is detected to be idle, the CRN will be allowed to transmit data; otherwise, the CRN will not be allowed to use the spectrum.

A synchronous system is assumed here, and the time is divided into slots, each with a fixed length $T$. The structure of the time slot is shown in Figure 1, where $\tau_{s}$ denotes the sensing time, and $\tau_{r}$ represents the reporting time of single $\mathrm{SU}$, and $T_{t}$ is the transmission time. In the reporting block, the local sensing results are reported to the fusion center through the common control channel (see [16]) sequentially. The common control channel operates at a different frequency than the PU, so there is no interference during the transmission of the reports.

Besides its low cost and simple hardware, the energy detector has been proved as the optimal method for spectrum sensing when prior information is unavailable [17]. Therefore, in this paper, we perform cooperatively spectrum sensing based on energy detection method in the CRN. The PU's signal is assumed to be phase-shift keying (PSK) signal; the noise is assumed to be additive white gauss noise (AWGN) with zero mean and variance $\sigma^{2}$, namely $N\left(0, \sigma^{2}\right)$. The test statistic for energy detector is given by $Y=\frac{\sum_{1}^{N}|y(n)|^{2}}{\sigma_{u}^{2}}$, where $N=\tau_{s} f_{s}$. To the $i$ th SU, the probabilities of false alarm and detection for the channel are given by [18]

$$
\begin{gathered}
P_{f}\left(\tau_{s}, \varepsilon_{i}\right)=Q\left(\frac{\varepsilon_{i}-\tau_{s} f_{s}}{\sqrt{2 \tau_{s} f_{s}}}\right) \\
P_{d}\left(\tau_{s}, \varepsilon_{i}\right)=Q\left(\frac{\varepsilon_{i}-\tau_{s} f_{s}-\gamma_{i}}{\sqrt{2 \tau_{s} f_{s}+4 \gamma_{i}}}\right)
\end{gathered}
$$

respectively, where $\tau_{s}$ represents the sensing time, $\varepsilon_{i}$ denotes the sensing threshold of energy detector of the $i$ th SU, $\gamma_{i}$ is the received signal-to-noise ratio (SNR) from the PU's signal measured at the $i$ th secondary receiver and $\gamma_{i}=N \gamma\left(\gamma\right.$ is defined in [18]), $f_{s}$ represents the sampling frequency, and finally, $Q(\cdot)$ is the complementary distribution function of standard Gaussian. Similar to $[18,19]$, we assume that the size of the CRN is small compared with its distance from the primary system. Therefore, the received signal at each $\mathrm{SU}$ experiences almost identical path loss. 


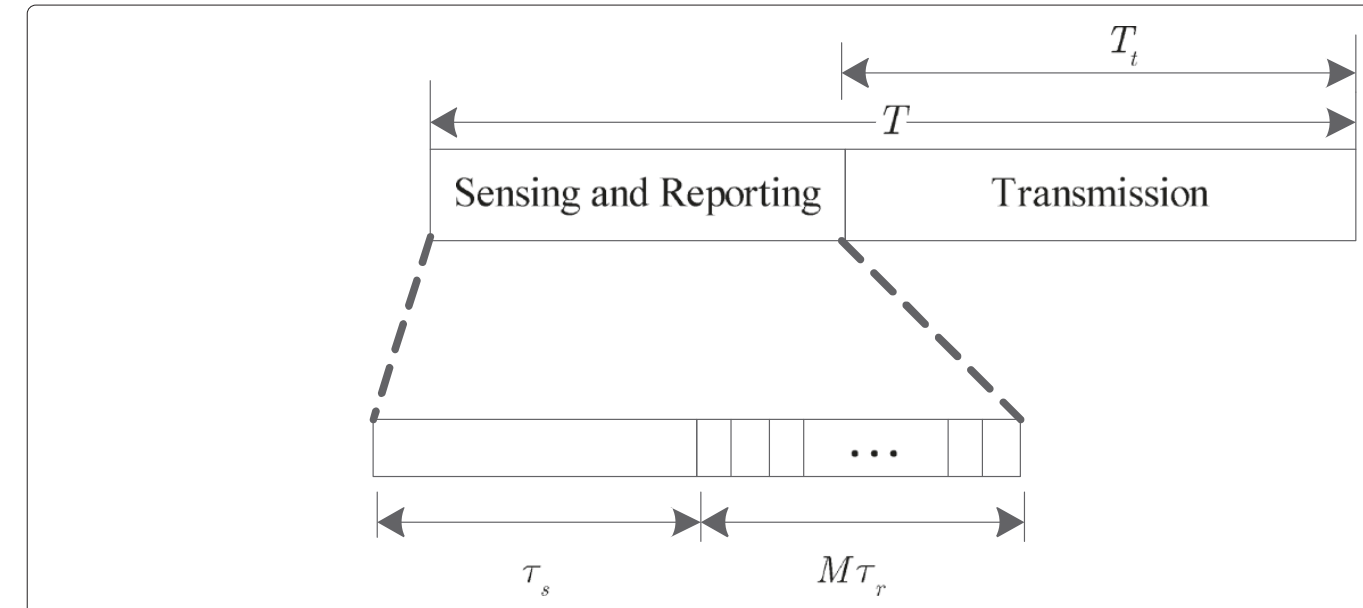

Figure 1 Structure of the time slot.

For simplicity, we assume that AND fusion rule is adopted here [18], where all of SUs' decisions say that the channel is active, then the final decision declares that the channel is active. By setting a common threshold $\varepsilon$ for all SUs, the overall probabilities of false alarm and detection for the channel are, respectively, given by [19]

$$
\begin{gathered}
Q_{f}\left(\tau_{s}, \varepsilon, M\right)=P_{f}\left(\tau_{s}, \varepsilon\right)^{M} \\
Q_{d}\left(\tau_{s}, \varepsilon, M\right)=P_{d}\left(\tau_{s}, \varepsilon\right)^{M}
\end{gathered}
$$

\section{Problem formulation}

\section{Average energy consumption of the CRN}

In our model, the total power consumption consists of four parts: circuit consumption $P_{c}$, spectrum sensing $P_{s}$, reporting $P_{r}$, and data transmission $P_{t}$. In the CRN, there are four different scenarios between the activities of the PU and the SUs.

S1: In this scenario, the SUs successfully detect the channel's idle status with probability $P_{0}\left(1-Q_{f}\left(\tau_{s}, \varepsilon, M\right)\right)$, where $P_{0}$ denotes the idle probability of the channel. And the energy consumed is given by

$$
\begin{aligned}
C_{1}\left(\tau_{s}, M\right)= & M\left(P_{s}+P_{c}\right) \tau_{s}+M\left(P_{r}+P_{c}\right) \tau_{r} \\
& +\left(T-\tau_{s}-M \tau_{r}\right)\left(P_{t}+P_{c}\right)
\end{aligned}
$$

S2: For this scenario, the SUs falsely detect the channel's idle status with probability $P_{0} Q_{f}\left(\tau_{s}, \varepsilon, M\right)$, and the energy used is given by

$$
C_{2}\left(\tau_{s}, M\right)=M\left(P_{s}+P_{c}\right) \tau_{s}+M\left(P_{r}+P_{c}\right) \tau_{r}
$$

S3: In this case, the SUs falsely detect the channel's active status with probability $P_{1}\left(1-Q_{d}\left(\tau_{s}, \varepsilon, M\right)\right)$, where
$P_{1}=1-P_{0}$ denotes the active probability of the channel, and the energy used is given by

$$
C_{3}\left(\tau_{s}, M\right)=C_{1}\left(\tau_{s}, M\right)
$$

S4: Under this scenario, the SUs successfully detect the channel's active status with probability $P_{1} Q_{d}\left(\tau_{s}, \varepsilon, M\right)$, and the energy consumed is given by

$$
C_{4}\left(\tau_{s}, M\right)=C_{2}\left(\tau_{s}, M\right)
$$

Hence, the average energy consumption in a time slot $T$ is approximated as

$$
\begin{aligned}
\psi\left(\tau_{s}, \varepsilon, M\right)= & x M \tau_{s}+y M+z\left(T-\tau_{s}-M \tau_{r}\right) \\
& \cdot\left(1-P_{0} Q_{f}\left(\tau_{s}, \varepsilon, M\right)-P_{1} Q_{d}\left(\tau_{s}, \varepsilon, M\right)\right)
\end{aligned}
$$

where $x=P_{s}+P_{c}, y=\left(P_{r}+P_{c}\right) \tau_{r}, z=P_{t}+P_{c}$.

\section{Average achievable data rate of the CRN}

A channel's availability for SU transmission is based on the outcome of the spectrum sensing. In case S3, the channel is detected to be idle while the channel is actually active, the PU's signal is an interference to SUs' transmission, and we assume the transmission will not succeed. Hence, only in case $S 1$ the data can be transmitted successfully.

Denote $C$ as the achievable data rate of the CRN when it operates in the absence of PU, and the value of $C$ is assumed to be [18]

$$
C=B \log _{2}\left(1+\mathrm{SNR}_{s}\right)
$$


where B is the bandwidth and $\mathrm{SNR}_{s}$ is the SNR for secondary transmission.

Hence, the average throughput of the CRN in a time slot $T$ can be expressed as

$$
W\left(\tau_{s}, \varepsilon, M\right)=J\left(T-\tau_{s}-M \tau_{r}\right)\left(1-Q_{f}\left(\tau_{s}, \varepsilon, M\right)\right)
$$

where $J=P_{0} C$.

\section{Energy utility function}

When the sensing time $\tau_{s}$ increases, the detection probability can be increased which can protect the PU. On the other hand, the energy consumed in the sensing phase also increases.

The increase of the number of cooperative SUs leads to a high space diversity gain and helps to improve the spectrum sensing performance. However, it also results in the increase of total reporting delay which leads to the decrease of the spectrum sensing time and the data transmission time. And more energy is used in the sensing phase. Hence, there are tradeoffs among the sensing performance, the achievable data rate, and the energy consumption when designing the sensing time and the number of cooperative SUs.

The utility function in [13] essentially accounts for the difference between a term related with date rate and a term related with energy consumption. In some cases, when the energy consumption terms are very small relative to the data rate, optimization on the difference may be influenced heavily by the data rate term. Such problems will be avoided by optimizing the ratio utility. In this paper, we propose an energy utility function, which is defined as

$$
\eta\left(\tau_{s}, \varepsilon, M\right)=\frac{W\left(\tau_{s}, \varepsilon, M\right)}{\left(\psi\left(\tau_{s}, \varepsilon, M\right)\right)^{v}}
$$

where $v$ with $v>0$ is the weighting factor denoting the weight of the energy consumption. The larger value of the weighting factor implies that the energy consumption is more important than the achievable data rate of the CRN. Specially, when $v=1,(12)$ denotes the achievable data rate per unit of energy.

For a given $v$, our objective is to maximize the energy utility function $\eta\left(\tau_{s}, \varepsilon, M\right)$, which suggests that the SUs try to use less energy to obtain more data rate. Therefore, an optimization problem of jointly designing the sensing time, the sensing threshold, and the number of cooperative SUs is formulated subject to the spectrum sensing accuracy, as shown by the following:

$$
\max _{\tau_{s}, \varepsilon, M} \eta\left(\tau_{s}, \varepsilon, M\right)=\frac{W\left(\tau_{s}, \varepsilon, M\right)}{\left(\psi\left(\tau_{s}, \varepsilon, M\right)\right)^{v}}
$$

subject to

$$
\begin{aligned}
& Q_{d}\left(\tau_{s}, \varepsilon, M\right)=\alpha \\
& 0<\tau_{s}<T \\
& 1 \leq M \leq N, M \in \mathbb{N}
\end{aligned}
$$

where $\alpha$ is the target detection probability to protect the PU.

\section{Solution of formulation}

Instead of solving the problem (13) directly, we propose the algorithm that solves the problem by an exhaustive search for $M$. Since $M$ is an integer and lies within the interval $[1, \mathrm{~N}]$, the computational complexity to search the optimal $\mathrm{M}$ is not very high.

In order to solve problem (13), we transform the problem to

$$
\max _{M} \eta(M)=\eta^{*}(M)
$$

subject to

$$
1 \leq M \leq N, M \in \mathbb{N}
$$

where the $\eta^{*}(M)$ is the optimal objective value of the following problem (15) with a specific $M$ value.

$$
\max _{\tau_{s}, \varepsilon} \eta\left(\tau_{s}, \varepsilon\right)=\frac{W\left(\tau_{s}, \varepsilon, M\right)}{\left(\psi\left(\tau_{s}, \varepsilon, M\right)\right)^{v}}
$$

subject to

$$
\begin{aligned}
& Q_{d}\left(\tau_{s}, \varepsilon, M\right)=\alpha \\
& 0<\tau_{s}<T
\end{aligned}
$$

We focus on $0<v \leq 1$; next, we discuss the solution of problem (15) under $v=1$ and $0<v<1$.

\section{Weighting factor $v=1$}

With the constraint $Q_{d}\left(\tau_{s}, \varepsilon\right)=\alpha$, we can obtain

$$
P_{d}\left(\tau_{s}, \varepsilon\right)=\alpha^{1 / M}
$$

Then we can get [18]

$$
P_{f}\left(\tau_{s}\right)=Q\left(u+h \sqrt{\tau_{s}}\right)
$$

where $u=\sqrt{1+2 \gamma} Q^{-1}\left(P_{d}\left(\tau_{s}, \varepsilon\right)\right), h=\gamma \sqrt{f_{s} / 2}$. With $P_{f}\left(\tau_{s}\right)$, we can get the $Q_{f}\left(\tau_{s}\right)$ through (3).

In a practical CRN, we set $\tau_{s}>(u / h)^{2}$, such that $P_{f}\left(\tau_{s}\right)<0.5[11]$.

Finally, problem (15) with $v=1$ is simplified to

$$
\max _{\tau_{s}} \eta\left(\tau_{s}\right)=\frac{W\left(\tau_{s}\right)}{\psi\left(\tau_{s}\right)}
$$

subject to

$$
(u / h)^{2}<\tau_{s}<T-M \tau_{r}
$$


The methodology used in [14] to solve the fractional optimization can be used here to solve the problem (18). We define a function expressed as

$$
f\left(\tau_{s}, \lambda\right)=W\left(\tau_{s}\right)-\lambda \psi\left(\tau_{s}\right)
$$

where $\lambda$ is an arbitrary positive number $[20,21]$. We define another function expressed as

$$
g(\lambda)=\max _{\tau_{s}} f\left(\tau_{s}, \lambda\right)
$$

If $g(\lambda)$ is a monotonically decreasing function in terms of $\lambda$, then the optimal solution of (18) occurs at $g(\lambda)=0$ [14].

Proof. It is easy to show that $g(\lambda)$ is a monotonically decreasing function in terms of $\lambda$. Since for any $\tau_{s}, \psi\left(\tau_{s}\right)>$ 0 . Hence, for any given $\tau_{s}, f\left(\tau_{s}, \lambda\right)$ must decrease as $\lambda$ increases. Next, we have

$$
g(\lambda)=\max _{\tau_{s}} f\left(\tau_{s}, \lambda\right) \geq f\left(\tau_{s}, \lambda\right)>f\left(\tau_{s}, \lambda+\delta\right), \forall \tau_{s}
$$

where the $\delta$ is any positive number. Therefore,

$$
g(\lambda)>\max _{\tau_{s}} f\left(\tau_{s}, \lambda+\delta\right)=g(\lambda+\delta)
$$

which proves that $g(\lambda)$ is a monotonically decreasing function in terms of $\lambda$.

For a given $\tau_{s}, f\left(\tau_{s}, \eta\left(\tau_{s}\right)\right)=0$. Set $\tau_{s}{ }^{*}$ as the optimal solution of (18), then $\lambda=\eta\left(\tau_{s}^{*}\right)$ is the largest $\lambda$ value that can satisfy $f\left(\tau_{s}, \lambda\right)=0$ since $\eta\left(\tau_{s}{ }^{*}\right)$ is the maximum value for any $\tau_{s}$. Next, we denote $\bar{\lambda}$ as $g(\bar{\lambda})=0$ and $\bar{\tau}_{s}$ as its corresponding $\tau_{s}$ value that can maximize $f\left(\tau_{s}, \bar{\lambda}\right)$. Any $\tilde{\lambda}>\bar{\lambda}$, $g(\tilde{\lambda})<0$ due to monotonicity. Hence, $\bar{\lambda}$ is the largest $\lambda$ value that can satisfy $f\left(\tau_{s}, \lambda\right)=0$. Therefore, $\bar{\lambda}$ must be equal to $\bar{\lambda}=\eta\left(\tau_{s}{ }^{*}\right)$, and $\bar{\tau}_{s}=\tau_{s}{ }^{*}$.

Due to the monotonicity of $g(\lambda)$, the optimal value $\eta\left(\tau_{s}{ }^{*}\right)$ which occurs at $g(\lambda)=0$ can be found by using the bisection algorithm.

Furthermore, when $\lambda$ satisfies $g(\lambda) \geq 0, f\left(\tau_{s}, \lambda\right)$ is a convex function when $\tau_{s}$ is in the given interval $\left((u / v)^{2}, T-M \tau_{r}\right)$.

Proof. Differentiating $f\left(\tau_{s}, \lambda\right)$ with respect to $\tau_{s}$, we get

$$
\begin{aligned}
f^{\prime}\left(\tau_{s}, \lambda\right)= & -J-\lambda x+\lambda z-\lambda z P_{1} \alpha+\left(J-\lambda z P_{0}\right) Q_{f}\left(\tau_{s}\right) \\
& -\left(J-\lambda z P_{0}\right)\left(T-\tau_{s}-M \tau_{r}\right) Q_{f}^{\prime}\left(\tau_{s}\right)
\end{aligned}
$$

Again differentiating $f^{\prime}\left(\tau_{s}, \lambda\right)$ with respect to $\tau_{s}$,

$$
\begin{aligned}
f^{\prime \prime}\left(\tau_{s}, \lambda\right)= & 2\left(J-\lambda z P_{0}\right) Q_{f}^{\prime}\left(\tau_{s}\right) \\
& -\left(J-\lambda z P_{0}\right)\left(T-\tau_{s}-M \tau_{r}\right) Q_{f}^{\prime \prime}\left(\tau_{s}\right)
\end{aligned}
$$

$P_{f}\left(\tau_{s}\right)$ is decreasing and convex with respect to $\tau_{s}$ when satisfying $P_{f}\left(\tau_{s}\right)<0.5$ [18]. Hence, there is $P_{f}^{\prime}\left(\tau_{s}\right)<0$, $P_{f}^{\prime \prime}\left(\tau_{s}\right)>0$, then we can get

$$
Q_{f}^{\prime}\left(\tau_{s}\right)=M P_{f}^{M-1}\left(\tau_{s}\right) P_{f}^{\prime}\left(\tau_{s}\right)<0
$$

$$
\begin{aligned}
Q_{f}^{\prime \prime}\left(\tau_{s}\right)= & M(M-1) P_{f}^{M-2}\left(\tau_{s}\right) P_{f}^{\prime 2}\left(\tau_{s}\right) \\
& +M P_{f}^{M-1}\left(\tau_{s}\right) P_{f}^{\prime \prime}\left(\tau_{s}\right)>0
\end{aligned}
$$

As $g(\lambda) \geq 0$, so $J-\lambda z P_{0}>0$, and there is

$$
f^{\prime \prime}\left(\tau_{s}, \lambda\right)<0
$$

For a given $\lambda$ satisfying $g(\lambda) \geq 0, f\left(\tau_{s}, \lambda\right)$ is a convex function with respect to $\tau_{s}$. And the optimal $\tau_{s}$ maximizing $f\left(\tau_{s}, \lambda\right)$ can be obtained by solving $f^{\prime}\left(\tau_{s}, \lambda\right)=0$. When $\lambda$ satisfying $g(\lambda)<0$, there is no need to find the optimal $\tau_{s}$. As we want to find the $\lambda$ satisfying $g(\lambda)=0$, however, for any $\tau_{s}$, there is $g(\lambda)<0$ in this case.

With a given number of cooperative SUs $M$ under the weighting factor $v=1$, the optimal sensing time can be obtained by Algorithm 1.

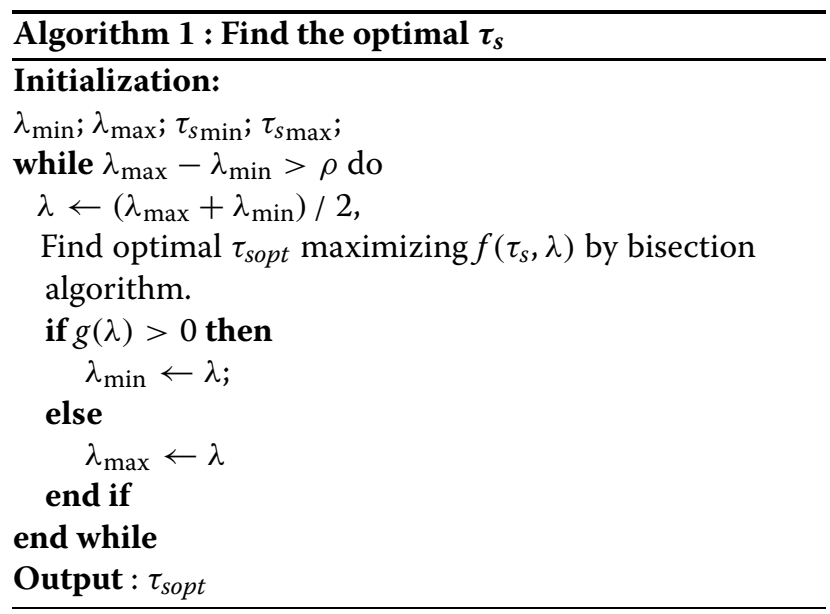

\section{Weighting factor $0<v<1$}

For a given $M$, similarly to the case $v=1$, the problem (15) can also be simplified to

$$
\max _{\tau_{s}} \eta\left(\tau_{s}\right)=\frac{W\left(\tau_{s}\right)}{\left(\psi\left(\tau_{s}\right)\right)^{v}}
$$

subject to

$$
0<\tau_{s}<T-M \tau_{r}
$$

In general, the $\eta\left(\tau_{s}\right)$ may not be a concave function in terms of $\tau_{s}$. Hence, we have to exhaustively search for the 
optimal sensing time over $\left(0, T-M \tau_{r}\right)$. Fortunately, from the numerical results, we can see that the optimal $\tau_{s}$ can be found by using bisection algorithm, which can decrease the complexity to find the solution of problem (25).

\section{Numerical results}

In this section, numerical results of the design will be presented. The default parameters are set as follows: the fixed time slot is set to be $T=20 \mathrm{~ms}$, the sampling frequency of the received signal is assumed to be $6 \mathrm{MHz}$, and the reporting time of single $\mathrm{SU}$ is set to be $\tau_{r}=$ $0.1 \mathrm{~ms}$. The SNR of the PU's signal received at the SUs is set to be $-20 \mathrm{~dB}$. The active probability of the channel is assumed to be $P_{1}=0.1$, and the threshold of the detection probability is $\alpha=0.95$. The secondary transmission $\mathrm{SNR}_{\mathrm{s}}=20 \mathrm{~dB}$. The weighting factor is set as $v=0.4$. Both the sensing and circuit power are set as $0.1 \mathrm{~W}$, and both the reporting and transmission power are assumed to be $3 \mathrm{~W}$ [12]. SNR referred in the following paper is the received signal-to-noise ratio from the PU at the secondary detector.

Figure 2 shows the energy utility versus the spectrum sensing time with different numbers of the cooperative SUs. It can be seen from this figure that, with the increase of the sensing time, the values of the energy utility in all curves first increase and then decrease. This indicates that an optimal sensing time can be obtained to maximize the energy utility when given the number of cooperative SUs. In Figure 2, the energy utility vs. the spectrum sensing time is compared among different numbers of cooperative SUs. We can see that with a given number of cooperative SUs, there is an optimal sensing time that can maximize the energy utility.

Figure 3 shows the energy utility vs. the number of cooperative SUs with different sensing times. Similar to Figure 2, with the increase of the number of cooperative SUs, the values of the energy utility in all curves first increase and then decrease. Therefore, there is an optimal number of cooperative SUs maximizing the energy utility under both the optimal sensing time and the fixed sensing time. Moreover, it can be observed that the energy utility with the optimal sensing time outperforms that with the fixed sensing time.

The optimal number of cooperative SUs, the optimal sensing time, and the energy utility vs. SNR are compared among different detection probability in Figures 4, 5,6 , respectively, when jointly optimizing the number of cooperative SUs and the sensing time. Form Figure 4, we can see that the optimal number of cooperative SUs decreases with the increasing of SNR and increases as the detection probability $\alpha$ takes higher values. In Figure 5, we also can find that the optimal sensing time decreases with the increasing of SNR and increases as the detection

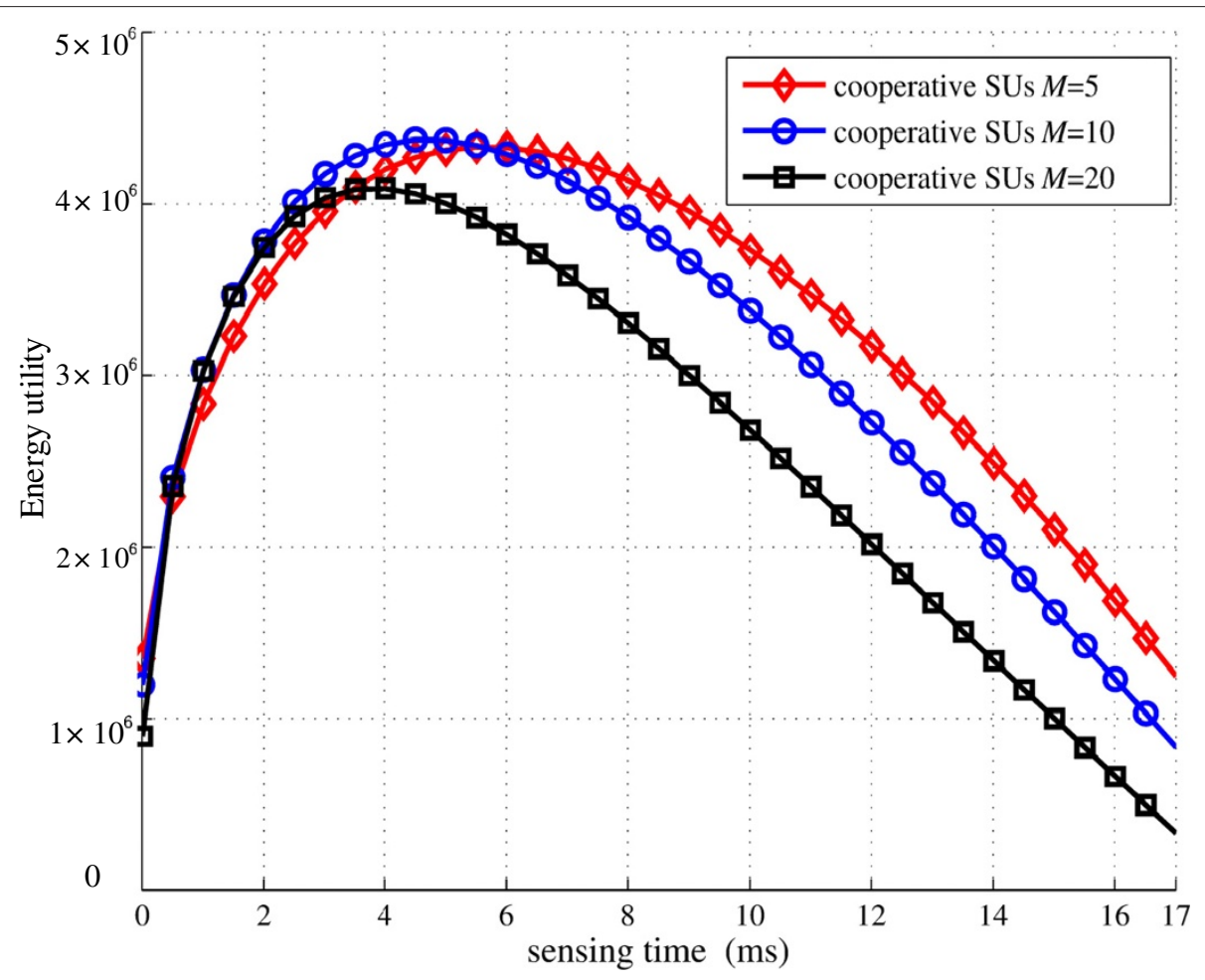

Figure 2 Energy utility vs. spectrum sensing time. 




Figure 3 Energy utility vs. numbers of cooperative SUs.

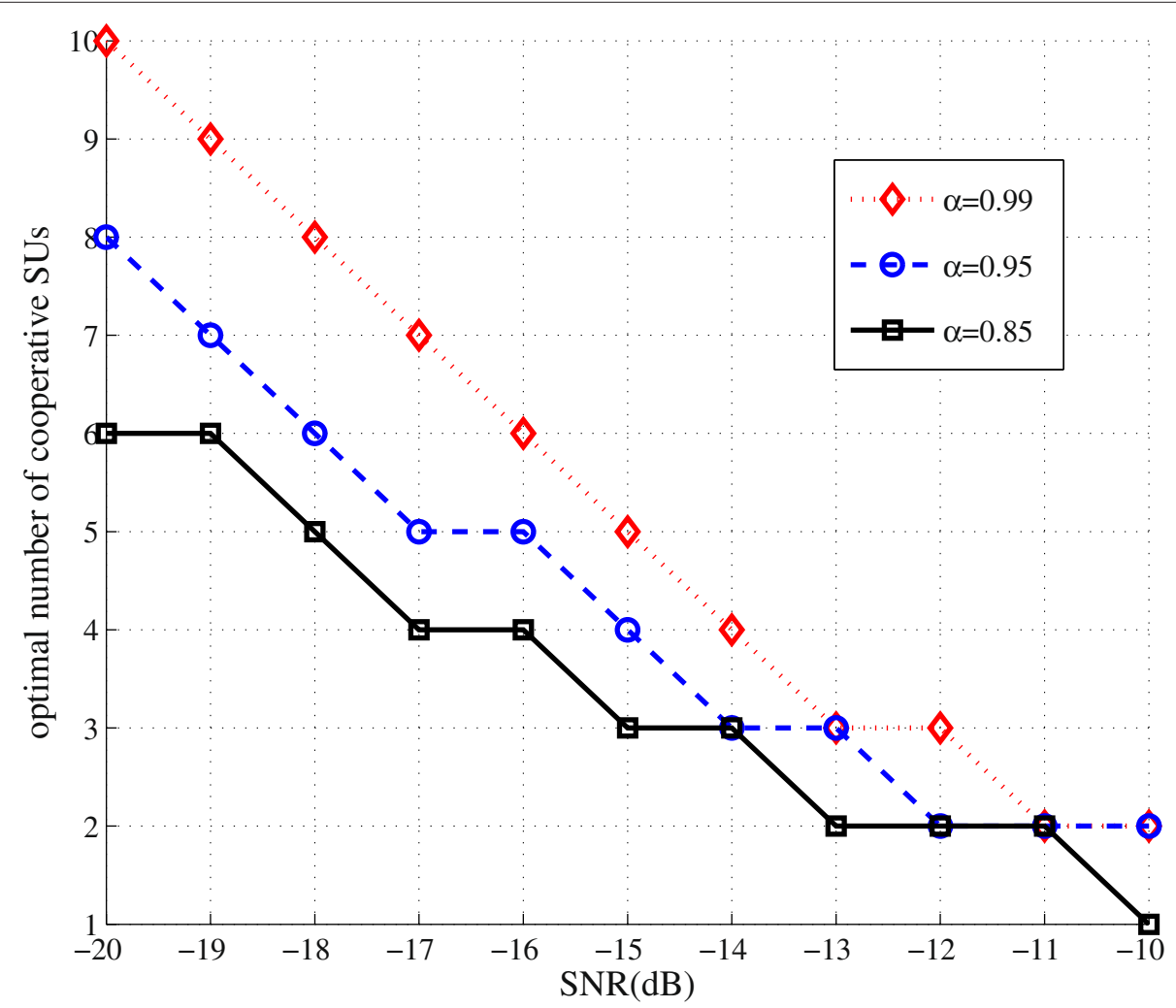

Figure 4 Optimal number of cooperative SUs vs. SNR. 


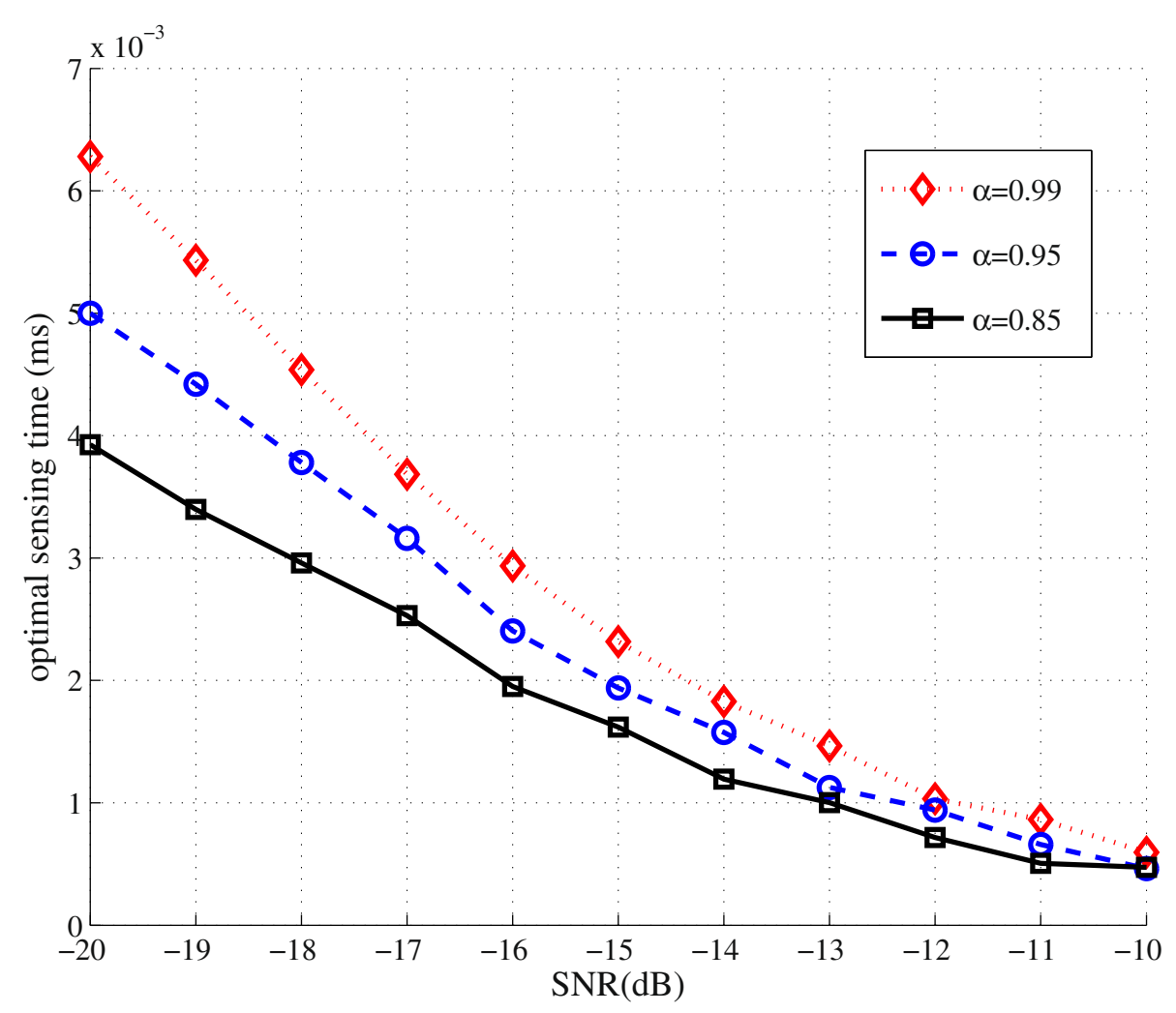

Figure 5 Optimal sensing time vs. SNR.

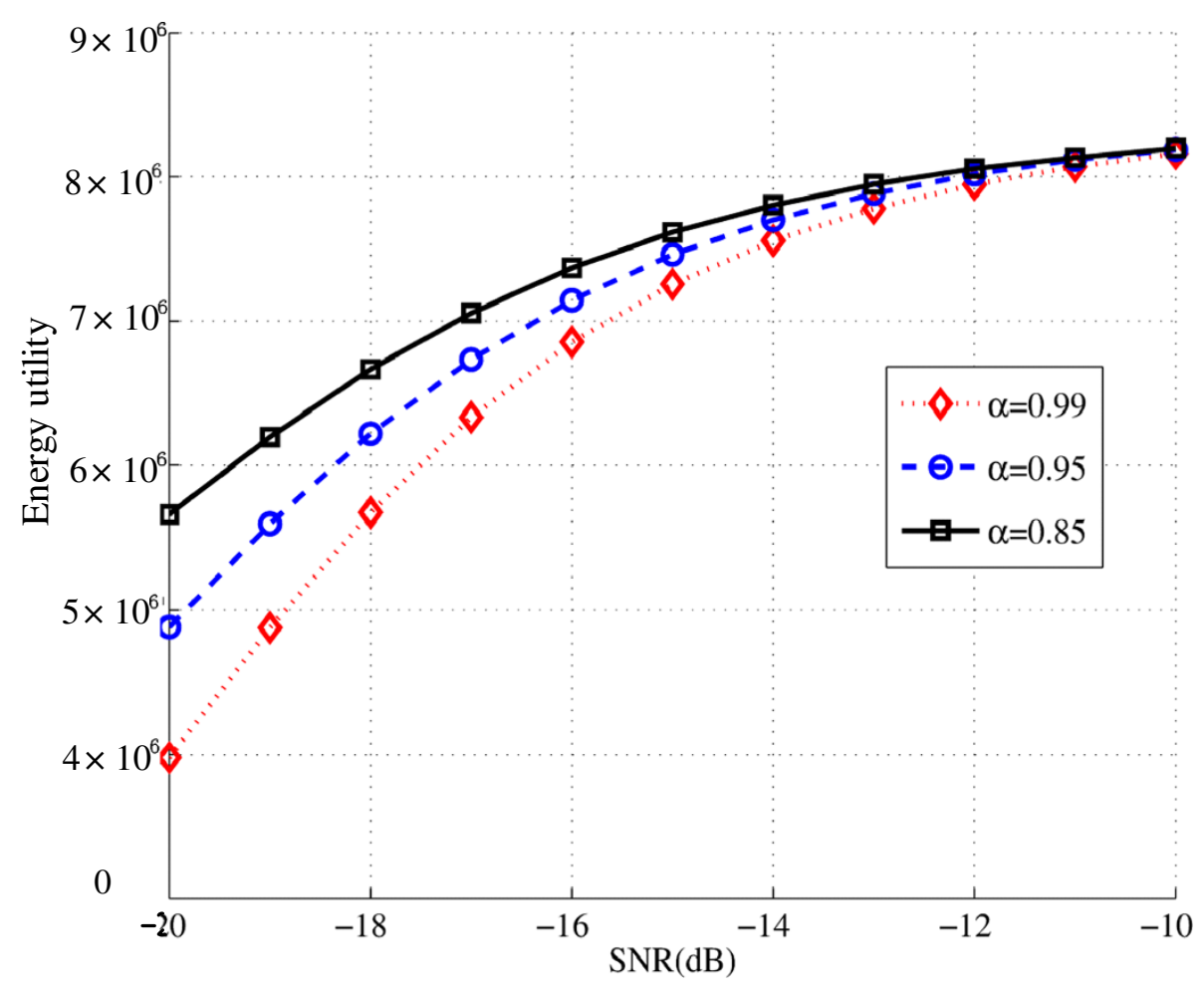

Figure 6 Energy utility vs. SNR. 


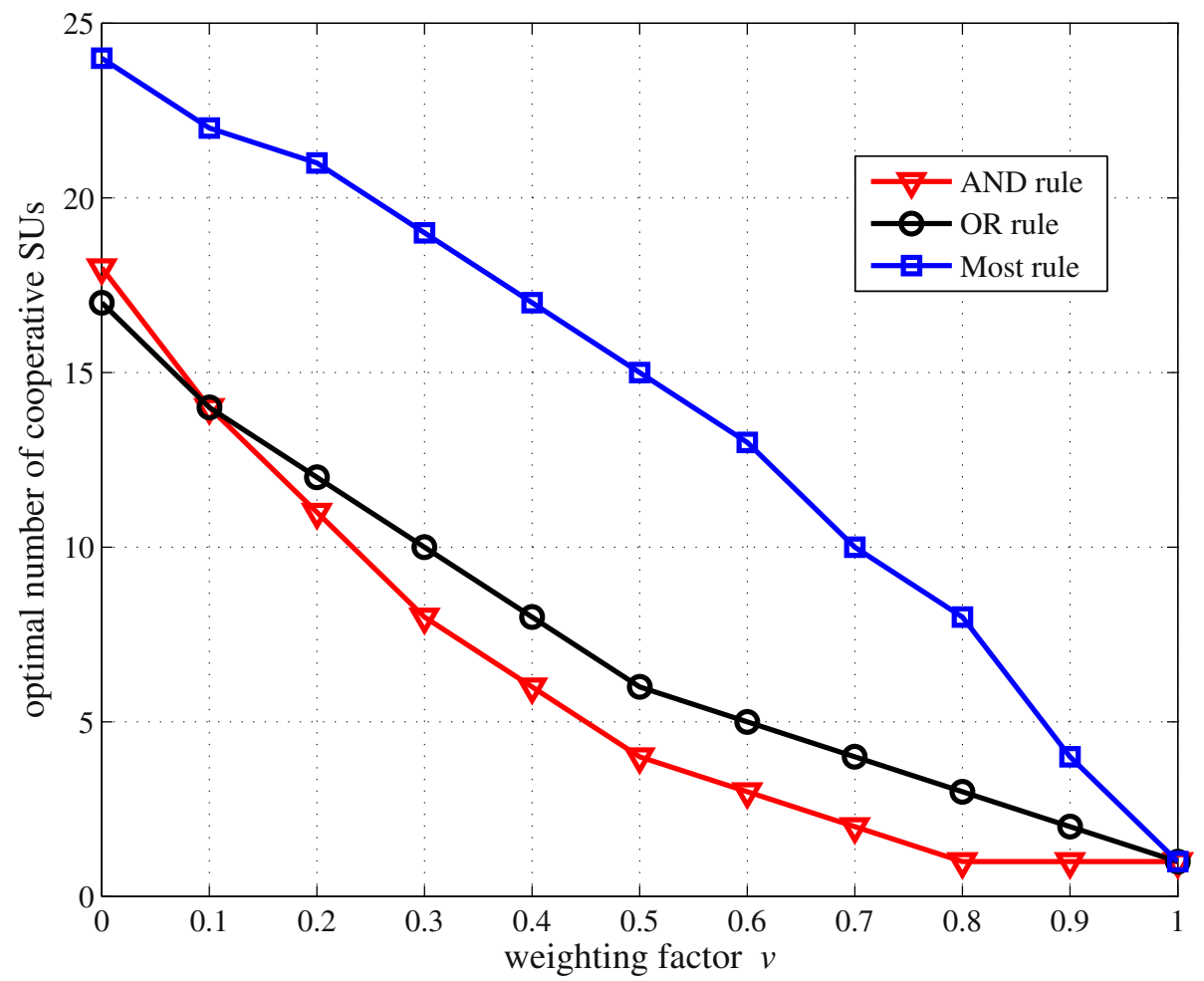

Figure 7 Optimal number of cooperative SUs vs. weighting factor.

probability $\alpha$ takes higher values. In Figure 6, when SNR increases, the energy utility also increases. This is because there is more transmission time and less energy consumption. However, the energy utility decreases with the increasing of detection probability $\alpha$.

In Figure 7, the optimal number of cooperative SUs vs. weighting factor is compared among different fusion rules. We can see that the optimal number of cooperative SUs under AND rule is the smaller than the other two fusion rules. When the weighting factor increases, the optimal number of cooperative SUs decreases because the increase of number of cooperative SUs would introduce more energy consumption. When the weighting factor is 1 , the optimal $M=1$, which indicates that the improvement in sensing performance is not able to outweigh the larger energy consumption introduced by the larger number of cooperative SUs when $v=1$.

\section{Conclusions}

In this paper, we propose an energy utility function by formulating the achievable data rate in terms of the energy consumption in a cooperative CRN. A weighting factor of energy consumption is introduced here. Taking the maximization of the energy utility as the design objective, optimization problem is formulated as a function of three variables, which are sensing time, sensing threshold, and the number of cooperative SUs, subject to the target detection probability. An algorithm is also presented to solve the problem, and numerical results show that the CRN can achieve almost the maximum achievable data rate with significant energy saving through the joint optimization.

\section{Competing interests}

The authors declare that they have no competing interests.

\section{Acknowledgements}

This work is supported by National Nature Science Foundation of China (Nos. $11301071,61372106,61102068,61172077,61223001)$, Key Special Project of National Science and Technology (No. 2013ZX03003006), National 863 High Technology Development Project (No. 2013AA013601), Research Fund of National Mobile Communications Research Laboratory, Southeast University (No. 2013A04, 2014B01), CPSF (2013M531244, 2014T70450), Research Fund for the Doctoral Program of Higher Education (20113218120017), and Open Research Fund of National Mobile Communications Research Laboratory, Southeast University (2012D14).

Received: 29 April 2014 Accepted: 29 September 2014 Published: 22 October 2014

\section{References}

1. A Ghasemi, ES Sousa, Spectrum sensing in cognitive radio networks: requirements, challenges and design trade-offs. IEEE Commun. Mag. 46(4), 32-39 (2008) 
2. J Mitola, GQ Maguire Jr., Cognitive radio: making software radios more personal. IEEE Personal Commun. 6(4), 13-18 (1999)

3. D Sahin, VC Gungor, T Kocak, G Tuna, Quality-of-service differentiation in single-path and multi-path routing for wireless sensor network-based smart grid applications, (2014). doi:10.1016/j.adhoc.2014.05.005

4. M Macit, VC Gungor, G Tuna, Comparison of QoS-aware single-path vs.multi-path routing protocols for image transmission in wireless multimedia sensor networks. Ad Hoc Netw. 19, 132-141 (2014)

5. G Tuna, VC Gungor, K Gulez, Wireless sensor networks for smart grid applications: a case study on link reliability and node lifetime evaluations in power distribution systems. Int. J. Distributed Sensor Netw. (Article ID 796248), 11 (2013). doi:10.1155/2013/796248

6. GA Shah, VC Gungor, OB Akan, A cross-layer QoS-aware communication framework in cognitive radio sensor networks for smart grid applications IEEE Trans. Ind. Inf. 9(3), 1477-1485 (2013)

7. VC Gungor, D Sahin, Cognitive radio networks for smart grid applications: a promising technology to overcome spectrum inefficiency. IEEE Vehicular Technol. Mag. 7(2), 41-46 (2012)

8. JY Wang, JB Wang, XY Song, M Chen, J Zhang, Network planning for distributed antenna-based high-speed railway mobile communications. Trans. Emerging Telecommun. Technol. 25(7), 707-722 (2014)

9. J-Y Wang, J-B Wang, M Chen, X Song, Performance analysis for free-space optical communications using parallel all-optical relays over composite channels. IET Commun. 8(9), 1437-1446 (2014)

10. IF Akyildiz, BF Lo, R Balakrishnan, Cooperative spectrum sensing in cognitive radio networks: a survey. Phys. Commun. 4(1), 40-62 (2011)

11. Z Shi, K Teh, KH Li, Energy-efficient joint design of sensing and transmission durations for protection of primary user in cognitive radio systems. Commun. Lett. 17(3), 565-568 (2013)

12. ECY Peh, YC Liang, YL Guan, YY Pei, in Proc. IEEE Global Telecommun. Conf. Energy-efficient cooperative spectrum sensing in cognitive radio networks (IEEE, Houston, 2011), pp. 1-5

13. XX Feng, XY Gan, XB Wang. Energy-constrained cooperative spectrum sensing in cognitive radio networks (IEEE, Houston, 2011), pp. 1-5

14. YY Pei, YC Liang, KC Teh, KH Li, Energy-efficient design of sequential channel sensing in cognitive radio networks: optimal sensing strategy, power allocation, and sensing order. IEEE J. Sel. Areas Commun. 29(8), 1648-1659 (2011)

15. S Althunibat, S Narayanan, MD Renzo, F Granelli, in IEEE International Workshop on Computer Aided Modeling and Design of Communication Links and Networks. On the energy consumption of the decision-fusion rules in cognitive radio networks (IEEE, Barcelona, 2012), pp. 125-129

16. F Gao, W Yuan, W Liu, WQ Cheng, S Wang, Pipelined cooperative spectrum sensing in cognitive radio networks. IEEE Wirel. Commun. Netw. Conf, 1-5 (2009)

17. FF Digham, MS Alouini, MK Simon, On the energy detection of unknown signals over fading channels. IEEE Trans. Commun. 55(1), 21-24 (2007)

18. YC Liang, YH Zeng, ECY Peh, AT Hoang, Sensing-throughput tradeoff for cognitive radio networks. IEEE Trans. Wirel. Commun. 7(4), 1326-1337 (2008)

19. ECY Peh, YC Liang, YL Guan, Optimization of cooperative sensing in cognitive radio networks: a sensing-throughput tradeoff view. IEEE Trans. Veh. Technol. 58(9), 5294-5299 (2009)

20. HL Zhu, JZ Wang, Chunk-based resource allocation in OFDMA systems part I: chunk allocation. IEEE Trans. Commun. 57(9), 2734-2744 (2009)

21. HL Zhu, JZ Wang, Chunk-based resource allocation in OFDMA systems part II: joint chunk, power and bit allocation. IEEE Trans. Commun. 60, 499-509 (2012)

doi:10.1186/1687-1499-2014-173

Cite this article as: Wu et al:: Optimal energy-efficient sensing in

cooperative cognitive radio networks. EURASIP Journal on Wireless

Communications and Networking 2014 2014:173.

\section{Submit your manuscript to a SpringerOpen ${ }^{\circ}$ journal and benefit from:}

- Convenient online submission

- Rigorous peer review

- Immediate publication on acceptance

- Open access: articles freely available online

- High visibility within the field

- Retaining the copyright to your article

Submit your next manuscript at $\gg$ springeropen.com 